\title{
Retrospektives Monitoring von Organozinnverbindungen in biologischen Proben aus Nord- und Ostsee - sind die Anwendungsbeschränkungen erfolgreich?
}

\author{
Heinz Rüdel · Jürgen Steinhanses · Josef Müller · Christa Schröter-Kermani
}

Erhalten: 16. Dezember 2008/Akzeptiert: 5. Januar 2009/Online veröffentlicht: 18. März 2009

(C) Springer-Verlag 2009

\begin{abstract}
Zusammenfassung Hintergrund, Ziel und Zweck Organozinnverbindungen werden als Biozide, Kunststoffadditive und Katalysatoren eingesetzt. Bezüglich der Umweltrelevanz am wichtigsten sind Tributylzinn- (TBT) und Triphenylzinnverbindungen (TPT), die bei Einträgen in Gewässer über eine hohe Toxizität verfügen und endokrine Wirkungen in Muscheln und Schnecken auslösen können. TBT wurde hauptsächlich als Antifouling-Wirkstoff in Schiffsanstrichmitteln eingesetzt. Diese Anwendung ist seit 1989 in Deutschland für Schiffe mit weniger als $25 \mathrm{~m}$ Länge untersagt. Seit 2003 ist in der Europäischen Union (EU) eine Richtlinie in Kraft, die die Anwendung von organozinnbasierten Antifouling-Anstrichen generell verbietet. Die hier vorgestellten Untersuchungen sollten überprüfen, ob die erlassenen Verbote zu einer Reduktion der Einträge in die marine Umwelt geführt haben.
\end{abstract}

Material und Methoden Für die Untersuchung wurden tiefgefrorene Homogenatproben von Miesmuscheln (Mytilus edulis) und Muskulatur von Aalmuttern (Zoarces viviparus) aus Nord- und Ostsee aus dem Archiv der Umweltprobenbank verwendet. Die Organozinnverbindungen wurden aus den biologischen Proben mit n-Hexan extrahiert und anschließend mit Natriumtetraethylborat derivatisiert. Nach

Prof. Dr. Dr. h. c. Volker Storch zum 65. Geburtstag gewidmet. Herausgeber: Ulrike Kammann · Henner Hollert

Gasteditoren: Gotthilf Hempel · Volker Storch

H. Rüdel $(\bowtie) \cdot J$. Steinhanses $\cdot$ J. Müller

Fraunhofer-Institut für Molekularbiologie

und Angewandte Oekologie (Fraunhofer IME),

Auf dem Aberg 1, 57392 Schmallenberg, Deutschland

E-Mail: heinz.ruedel@ime.fraunhofer.de

C. Schröter-Kermani

Umweltbundesamt, FG II 1.2,

Corrensplatz 1, 14195 Berlin, Deutschland kapillargaschromatografischer Trennung wurden die Derivate mit einem Atomemissionsdetektor quantifiziert.

Ergebnisse und Diskussion Zusammen mit einer früheren Untersuchung (Rüdel et al. 2003) umfassten die Zeitreihen Miesmuschel- und Fischmuskulaturproben der Jahre 1985 bis 2006. Die Daten zeigen, dass die TBT-Gehalte bis Ende der 1990er-Jahre unverändert blieben (z. B. in Miesmuscheln aus dem Jadebusen/Nordsee: $17 \pm 3 \mathrm{ng} / \mathrm{g}$ Frischgewicht, FG). Offensichtlich zeigte das seit 1989 in Deutschland geltende Verbot der TBT-Anwendung bei kleinen Schiffen in dieser Meeresregion keine Wirkung, da hier der Verkehr mit großen Schiffen dominiert. Der weitere Verlauf der Zeitreihen belegt jedoch, dass die TBT-Konzentrationen in Miesmuscheln und Aalmuttern nach 2003, als die EU-Richtlinie zum generellen Verbot der Organozinnverbindungen in Kraft trat, signifikant abnehmen. 2004 und 2005 wurden in den Muscheln aus dem Jadebusen nur noch TBT-Gehalte von 14 bzw. 6 ng/g FG gefunden. In Aalmuttern aus derselben Region sanken die Gehalte an TBT zwischen Ende der 1990erJahre und 2006 auf ca. 30\% des Ausgangswertes. Auch für TPT, das zeitweise ebenfalls als Antifouling-Wirkstoff eingesetzt wurde, sind deutliche Abnahmen in Muscheln und Fischen zu beobachten. Der statistisch signifikante Rückgang der OZV-Belastungen in den untersuchten Nordseeregionen wird durch Messungen in Muscheln und Fischen von einem küstennahen Ostseestandort bestätigt.

Schlussfolgerungen Insgesamt belegen die Untersuchungen den Erfolg der regulatorischer Maßnahmen zur Minderung der Einträge von Organozinnverbindungen in die aquatische Umwelt. Trotz der Reduktion zeigen die Gewebekonzentrationen aber auch, dass OZV nach wie vor Relevanz als marine Schadstoffe haben. Eine Umrechnung der Gewebekonzentrationen auf Wasserkonzentrationen ergibt, dass diese noch über der im Kontext der Wasserrahmenrichtlinie abgeleiteten Umweltqualitätsnorm von $0,2 \mathrm{ng} / \mathrm{l}$ liegen. Auch 
von OSPAR publizierte Bewertungskriterien (Environmental Assessment Criteria, EAC; 2,4 ng/g FG) werden aktuell noch überschritten. Insofern sind schädliche Wirkungen auf marine Organismen durch TBT nicht auszuschließen.

Empfehlungen und Ausblick Weitere Untersuchungen sollen zeigen, ob die abnehmenden Trends andauern. Hierzu sollte eine empfindlichere Methode wie z.B. speziesspezifische Isotopenverdünnungsanalytik verwendet werden, um niedrigere Bestimmungsgrenzen $\mathrm{zu}$ erreichen und die inzwischen abgesunkenen Konzentrationen mit ausreichender Sicherheit quantifizieren zu können.

Schlüsselworter Aalmutter · Antifouling ·

Chemikalienverbot · Miesmuscheln · Marines Monitoring · Mytilus edulis $\cdot$ Nordsee $\cdot$ Ostsee $\cdot$

Retrospektives Monitoring · TBT · Tributylzinn · TPT

Triphenylzinn · Umweltmonitoring · Umweltprobenbank ·

Zoarces viviparus

\section{Retrospective monitoring of organotin compounds in biological samples from North Sea and Baltic Sea - are the use restrictions successful?}

\begin{abstract}
Background, aim, and scope Organotin compounds are used as biocides, plastic additives and catalysts. With respect to environmental effects, tributyltin (TBT) and triphenyltin (TPT) compounds are the most relevant, because of their high aquatic toxicity and endocrine effects on mussels and snails. TBT was mainly used as antifouling agents in coatings of ships and boats. In 1989, Germany banned the application to ships $<25 \mathrm{~m}$ length. Finally, in 2003, the use of organotin-based antifoulants within the European Union was completely banned. To verify the effectiveness of the restrictions a retrospective monitoring study was initiated.

Material and methods A set of appropriate archived samples was retrieved from the German environmental specimen bank (ESB) comprising standardized pooled samples of eelpout (Zoarces viviparus) muscle tissue and of soft bodies of common mussels (Mytilus edulis) sampled at two locations in the North Sea and one in the Baltic Sea. Analysis of organotin compounds included n-hexane extraction, derivatisation with sodium tetraethyl borate, capillary gaschromatographic separation and atomic emission detection. Results and Discussion Altogether, time series cover the period 1985 to 2006, including data of an earlier study (Rüdel et al. 2003). Until the late 1990s, TBT remained more or less constant in all samples (e.g. $17 \pm 3 \mathrm{ng} / \mathrm{g}$ wet weight, ww, in mussels from Jadebay/North Sea). The German ban of TBT-based antifoulings for small ships had no effects on environmental concentrations because large ships dominate in the investigated North Sea regions. After the EU-wide ban of TBT in 2003, however, significant decreases in mus-
\end{abstract}

sel and fish contamination could be observed. In mussels from Jadebay, TBT concentrations were 14 and $6 \mathrm{ng} / \mathrm{g}$ ww in 2004 and 2005, respectively. TBT contamination in eelpout of the same region decreased to about 30 percent of the initial concentrations in 2006. Corresponding decreases were detected for TPT. Declining trends were also found in fish and mussels sampled from a Baltic Sea offshore site.

Conclusions The results demonstrate the effectiveness of the legal measures undertaken to control organotin inputs into the aquatic environment. Nevertheless, organotin compounds are still relevant pollutants. Water concentrations calculated from the measured tissue concentrations by using the respective bioconcentration factors are still above the Environmental Quality Standards derived in the context of the Water Framework Directive $(0.2 \mathrm{ng} / \mathrm{l})$ and the OSPAR mussel EAC (Environmental Assessment Criteria; $2.4 \mathrm{ng} / \mathrm{g} \mathrm{ww}$ ). Thus adverse effects to marine organisms cannot be excluded.

Recommendations and perspectives Further studies should be performed to verify the declining trends. More sensitive analytical methods, e.g. species-specific isotope dilution analysis, are recommended in order to detect lower environmental concentrations.

Keywords Antifouling · Baltic Sea · Blue mussels · Eelpout · Chemical ban · Environmental monitoring · Environmental specimen bank · Marine monitoring · Mytilus edulis · North Sea $\cdot$ Retrospective monitoring TBT $\cdot$ Tributyltin $\cdot$ TPT $\cdot$ Triphenyltin $\cdot$ Zoarces viviparus

\section{Hintergrund, Ziel und Zweck}

Organozinnverbindungen (OZV) haben seit Jahrzehnten eine große industrielle Bedeutung. Mitte der 1990er-Jahre lag der weltweite Verbrauch bei 40.000t pro Jahr (Graf 1996). OZV werden als Biozide (Wirkstoffe in AntifoulingAnstrichen für Schiffe, Holz- und Materialschutzmittel beispielsweise für Textilien und Leder, Pilzbekämpfungsmittel in der Landwirtschaft), als Wärme- und Lichtstabilisatoren für PVC-Kunststoffe, als Katalysatoren für spezielle Polyurethan-, Silikon- und Polyesterpolymere oder zur Vergütung von Glasoberflächen verwendet. Als Kunststoffstabilisatoren werden mono- und disubstituierte OZV eingesetzt (beispielsweise Mono- und Dioctylzinn- oder Mono- und Dibutylzinnverbindungen). OZV werden bei Gebrauch freigesetzt, können aber auch durch Emissionen aus der Produktion in die Umwelt gelangen. So lag zumindest in den 1990er-Jahren der weltweit bedeutendste Produktionsstandort für TBT im deutschen Rheineinzugsgebiet (IKSR 2002). Die Nutzung der Triorganozinnverbindungen als Biozide (ca. 18\% der Gesamtproduktion; Graf 1996) führt aber zu den mengenmäßig höchsten Einträgen in die Umwelt, da hier eine beabsichtigte Freisetzung erfolgt (z. B. in 
die aquatische Umwelt: kurzfristig aus dem Run-off beim Einsatz als Fungizid oder langfristig über die Lebensdauer des Anstrichs bei Antifouling-Beschichtungen). Die beiden wichtigsten Klassen für diese Anwendungen sind Tributylzinn- (Tributyltin, TBT) und Triphenylzinnverbindungen (Triphenyltin, TPT). Mitte der 1990er-Jahre wurden in Europa ca. 1.330 t/a OZV für Antifouling-Beschichtungen verbraucht, davon $<150 \mathrm{t} / \mathrm{a}$ in Deutschland (IKSR 2002). Im Wesentlichen handelte es sich hierbei um TBT-Verbindungen.

OZV sind mäßig persistent und in der Umwelt ubiquitär zu finden. Der Verbleib im aquatischen Ökosystem ist abhängig von relativ schnellen biotischen Abbauprozessen unter aeroben Bedingungen (z. B. durch Mikroorganismen) und langsamer Degradierung im anaeroben Milieu. Auch langsam verlaufende abiotische Reaktionen, wie Photolyse, und rasche, reversible Adsorptions- und Desorptionsprozesse an Feststoffen spielen eine Rolle. Die Bioverfügbarkeit für Organismen und die Ökotoxizität sind am höchsten im neutralen bzw. leicht alkalischen pH-Bereich. Die Anwesenheit von gelöstem organischem Kohlenstoff reduziert die Toxizität. Da die Aufnahme vorwiegend über die Wasserphase erfolgt, ist für rein aquatisch lebende Organismen die Biokonzentration aus der Wasserphase wichtiger als die Biomagnifikation über die Aufnahme OZV-belasteter Nahrung (Rüdel 2003).

Wegen ihrer toxischen Wirkung werden Organozinnverbindungen schon seit längerer Zeit intensiv untersucht (Fent 1996). Eine aktuelle Übersicht zu Untersuchungen der akuten Wirkungen zeigt Antizar-Ladislao (2008). Watermann et al. (2003) evaluierten endokrine Effekte von Tributylzinn auf Weichtiere und Säugetiere im Bereich der Nordsee. Hall et al. (2000) werteten alle für Salzwasserspezies verfügbaren Effektstudien aus und berechneten die 10er-Perzentile für Effekte bei den empfindlichsten Arten. Für akute Wirkungen ergab sich eine TBT-Konzentration von $320 \mathrm{ng} / \mathrm{l}$ und für chronische Effekte von $5 \mathrm{ng} / \mathrm{l}$. Triorganozinnverbindungen gelten wegen ihrer endokrinen Wirkung als besonders umweltrelevant. So führen TBT und TPT in Muscheln und Schnecken schon im ng/l-Bereich zu Sterilität (z.B. durch den Imposex-Effekt) und können damit eine verringerte Reproduktion und Bedrohung von Populationen zur Folge haben (Gibbs und Bryan 1996; Schulte-Oehlmann et al. 2000). Im Kontext der europäischen Wasserrahmenrichtlinie (EURichtlinie 2000/60/EG 2000) wurde TBT als prioritärer Stoff eingestuft und auf Basis der verfügbaren ökotoxikologischen Daten eine Umweltqualitätsnorm (UQN) in Wasser von $0,2 \mathrm{ng} / 1$ abgeleitet (EU-Kommission 2005).

Aufgrund der Hinweise auf die Ökotoxizität der OZV wurde schon 1989 die Anwendung von TBT für Schiffe mit Längen unter $25 \mathrm{~m}$ in den Mitgliedsstaaten der Europäischen Gemeinschaft untersagt. Aber erst seit 2003 ist die Nutzung von organozinnhaltigen Antifouling-Produkten durch die EU-Richtlinie 2002/62/EG insgesamt verboten (EU-Kommission 2002). TPT wurde in Deutschland noch bis zum Jahr 2001 als Wirkstoff in Fungiziden, beispielsweise im Kartoffelanbau, verwendet. Zudem wurde TPT in früheren Jahren als (zusätzlicher) Wirkstoff in AntifoulingBeschichtungen benutzt (IKSR 2002).

Zur Überprüfung, inwieweit die in der Vergangenheit getroffenen gesetzlichen Regelungen tatsächlich zu Rückgängen der Umweltkonzentrationen an OZV führen, wurde ein retrospektives Monitoring konzipiert (Arbeitskreis Umweltmonitoring 2007). Zu diesem Zweck wurde die Umweltprobenbank des Bundes (UPB) genutzt. Hierbei handelt es sich um ein Archiv von in regelmäßigen zeitlichen Abständen gesammelten repräsentativen Umweltproben (BMU 2000). Als Teil der ökologischen Umweltbeobachtung dient die UPB dazu, mögliche Fehlentwicklungen in Ökosystemen zu erkennen sowie Art und Umfang eventueller Schäden zu identifizieren. Außerdem kann die UPB Erkenntnisse für die Prioritätensetzung politischer Maßnahmen liefern und dazu beitragen, Grundlagen für die Vorsorgepolitik zu erarbeiten. Dabei können insbesondere solche Stoffe untersucht werden, die bei der Archivierung der Proben nicht als gefährlich angesehen wurden oder die zum Zeitpunkt der Probennahme noch nicht mit genügender Empfindlichkeit analysierbar waren. Beispiele für die Nutzung der UPB im Rahmen der Expositionsbewertung von Chemikalien sind retrospektive Analysen auf Alkylphenole und -ethoxylate (Wenzel et al. 2004), polycyclische Moschusverbindungen (Rüdel et al. 2006) oder Triclosan/Methyltriclosan (Böhmer et al. 2004).

Für OZV wurden im Rahmen der UPB bislang retrospektive Studien mit marinen Proben (Rüdel et al. 2003) und Süßwasserfischen (Rüdel et al. 2007) durchgeführt. Um eine frühere Untersuchung von Nord- und Ostseeorganismen zu ergänzen, die den Zeitraum 1985-1999 abdeckte (Rüdel et al. 2003), wurden Miesmuschel- und Aalmuttermuskulaturproben der Jahre 1999 bis 2006 (Aalmutter) bzw. 2000 bis 2005 (Miesmuschel) aus dem UPB-Archiv entnommen und für das retrospektive Monitoring eingesetzt.

\section{Material und Methoden}

Um für die UPB eine hohe Kontinuität zu sichern, sind alle Arbeitsschritte von der Probennahme über den Transport und die Aufbereitung zu Jahreshomogenaten bis zur Langzeitlagerung durch spezielle UPB-Verfahrensrichtlinien beschrieben (UBA 1996).

\subsection{Probenahme}

Die marinen Probenahmegebiete der UPB liegen in küstennahen Regionen von Nord- und Ostsee (BMU 2000). Als repräsentative Regionen des Wattenmeers wurden der Ja- 
debusen einschließlich Mellum (Nationalpark, NP, Niedersächsisches Wattenmeer) sowie die Meldorfer Bucht und das Sylt-Rømø-Watt (NP Schleswig-Holsteinisches Wattenmeer) gewählt. In der Ostsee liegen die Probenahmeflächen im NP Vorpommersche Boddenlandschaft vor der Halbinsel Darß/ Zingst. Für diese Studie wurden Proben von Aalmuttern (Zoarces viviparus) und Miesmuscheln (Mytilus edulis) eingesetzt. Aalmuttern werden im Juni nach der Laichzeit beprobt (Muskulatur und Leber), Miesmuscheln (Weichkörper) zweimonatlich (Nordsee) bzw. halbjährlich (Ostsee). Von jeder Probe werden jährlich 2-3 kg gesammelt und jeweils direkt vor Ort eingefroren (Klein et al. 1994). Die Biota-Probenahmen, bei der auch wichtige biometrische Parameter der Organismen erfasst werden, erfolgen durch Mitarbeiter des Instituts für Biogeographie der Universität Trier.

\subsection{Probenaufarbeitung}

Aus den tiefgefrorenen Proben werden Jahreshomogenate erstellt, indem das Probenmaterial bei Tiefsttemperaturen mit einer Cryomühle zerkleinert und homogenisiert wird (Koglin et al. 1997). Anschließend werden jeweils 200 Teilproben zu ca. $10 \mathrm{~g}$ abgefüllt. Die Lagerung erfolgt in der Gasphase über Flüssigstickstoff, wobei sich durch den verdampfenden Stickstoff eine inerte Gasatmosphäre bildet. Hierdurch sowie durch die niedrigen Temperaturen wird erreicht, dass die Proben über einen langen Zeitraum stabil sind. Zurzeit sind ca. 250 marine Proben mit mehr als 52.000 standardisierten UPB-Teilproben archiviert.

\subsection{Standards und OZV-Analysenmethode}

Analysen wurden durchgeführt wie bei Rüdel et al. (2003, 2007) beschrieben. Standards mit einer Reinheit zwischen 95 und 99\% wurden von ABCR/Strem, ACROS, Aldrich und Witco GmbH bezogen. TBT-Chlorid (CAS 1461-22-9), Dibutylzinndichlorid (DBT; CAS 683-18-1), Monobutylzinntrichlorid (MBT; CAS 1118-46-3), TPT-Chlorid (CAS 639-58-7), Diphenylzinndichlorid (DPT; CAS 1135-99-5), Monophenylzinntrichlorid (MPT; CAS 1124-19-2), Monooctylzinntrichlorid (MOT; CAS 3091-25-6) und Dioctylzinndichlorid (DOT; CAS 3542-36-7) wurden als OZV-Standards verwendet. Um die Vollständigkeit der Derivatisierung auf jedem Alkylierungsniveau überprüfen zu können, wurden Tripropylzinnchlorid, Diheptylzinndichlorid und Monoheptylzinntrichlorid als interne Standards verwendet. Alle benötigten Glasgeräte wurden vor Gebrauch bei $190^{\circ} \mathrm{C}$ ausgeheizt und nur für diese Anwendung benutzt. Für die Analytik wurde je $1 \mathrm{~g}$ der Miesmuschel- und Aalmuttermuskulaturproben mit

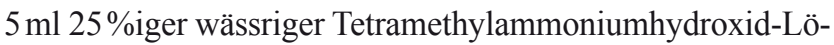
sung zunächst $1 \mathrm{~h}$ bei $60^{\circ} \mathrm{C}$ aufgelöst und mit einer Lösung der internen Standards versetzt. Nach dem Abkühlen wurden zu den aufgelösten Biotaproben $10 \mathrm{ml}$ Wasser, $5 \mathrm{ml}$ 10\%ige
Salzsäure und $20 \mathrm{ml}$ Na-Diethyldithiocarbamat-Lösung in Aceton (393 mg/l) sowie $20 \mathrm{ml} \mathrm{n-Hexan} \mathrm{gegeben,} \mathrm{um} \mathrm{die}$ enthaltenen OZV durch Schütteln in die organische Phase zu überführen. Die organische Phase wurde durch Dekantieren oder Zentrifugieren abgetrennt, mit $10 \mathrm{ml}$ Wasser, $10 \mathrm{ml}$ Ethanol, $2 \mathrm{ml}$ Acetatpuffer, $5 \mathrm{~g}$ Natriumchlorid und 0,5 ml Derivatisierungsmittel (5 g Natriumtetraethylborat, von ABCR/Strem oder GALAB, in $50 \mathrm{ml}$ Tetrahydrofuran) versetzt und der $\mathrm{pH}$ auf 4,5 eingestellt. Nach 30 min Schütteln wurde die Hexanphase abgenommen, mit ca. $2 \mathrm{~g}$ Natriumsulfat getrocknet und auf ca. 1-2 ml eingeengt. Der Extrakt wurde auf einer Silicagel-Säule ( $5 \mathrm{~g}$, überschichtet mit $2 \mathrm{~g}$ Natriumsulfat) gereinigt. Die Analyten wurden mit 5\% Aceton in Hexan eluiert, das Eluat auf $1 \mathrm{ml}$ eingeengt und die OZV-Derivate dann kapillargaschromatografisch getrennt (HP-5 Säule, $30 \mathrm{~m} \times 0,32 \mathrm{~mm}$; 0,25 $\mu \mathrm{m}$ Film, HP 5890 Series 2 GC). Quantifiziert wurde mit einem Atomemissionsdetektor (AED; HP 5921 oder HP 2350A; Messung der zinnspezifischen Wellenlängen $271 \mathrm{~nm}$ und $303 \mathrm{~nm}$ ). Die Einstellungen am GC waren: Trägergasdruck $85 \mathrm{kPa}$, Fluss $2,5 \mathrm{ml} / \mathrm{min}$, Anfangstemperatur $60^{\circ} \mathrm{C}$ für $1 \mathrm{~min}$, mit $10^{\circ} \mathrm{C} / \mathrm{min}$ auf $200^{\circ} \mathrm{C}, 6$ min halten, dann mit $20^{\circ} \mathrm{C} /$ min auf $300^{\circ} \mathrm{C}, 9 \mathrm{~min}$ Halten. Die Kalibrierung erfolgte über das Gesamtverfahren einschließlich Matrix. Die OZV-Konzentrationen werden als ng Organozinnkation (OZK) prog Frischgewicht (FG) angegeben.

\subsection{Qualitätssicherung}

Bei jeder Messserie wurden Leerwertproben sowie Referenzmaterialproben mitgeführt. Die Leerwertproben zeigten keine Hinweise auf eine mögliche Kontamination aus Reagenzien oder Geräten. Die mittleren OZV-Wiederfindungsergebnisse für das Verfahren ohne Matrix lagen im Bereich 88-107\% $(n=9-10)$. Als zertifiziertes Referenzmaterial (certified reference material, CRM) wurde ERM-CE 477 (Muschelgewebe) verwendet, das für Butylzinnverbindungen zertifiziert ist. Die Ergebnisse des jeweils parallel zu den UPB-Proben untersuchten CRM ergaben 1,87 $\pm 0,23 \mu \mathrm{g} / \mathrm{g}$ für TBT (Wiederfindung 84,9\%), 1,45 $\pm 0,35 \mu \mathrm{g} / \mathrm{g}$ für DBT $(93,9 \%)$ und $2,04 \pm 0,23 \mu \mathrm{g} / \mathrm{g}$ für MBT (136\%). Die Wiederfindungen für TBT und DBT sind zufriedenstellend, während die für MBT zu hoch liegt. Dieses Ergebnis zeigte sich schon in früheren Untersuchungen (Rüdel et al. 2003); allerdings ist auch die Unsicherheit des zertifizierten MBT-Gehalts mit ca. 20\% relativ hoch. Insgesamt liegt das Belastungsniveau des CRM deutlich oberhalb der Konzentrationen, die in Nord- und Ostseemuscheln zu finden sind ( $\mu \mathrm{g} / \mathrm{g}$ im Vergleich zu ng/g), sodass die Aussagekraft für die hier untersuchten Proben eingeschränkt ist. Deshalb wurden zusätzlich aufgestockte UPB-Proben mit niedrigeren OZV-Belastungen als laborinterne Referenzmaterialien verwendet (Miesmuscheln Eckwarderhörne 1990, Aalmuttermuskulatur Jadebusen 1997). Die mittleren Wiederfindungen für beide Matrices, berechnet 
als Gehalt vor Aufstockung im Verhältnis zur Probe inklusive Aufstockung in \%, betrugen: MBT $65 \pm 12 \%(n=10)$; DBT $72 \pm 13 \%(n=10)$; MPT $72 \pm 18 \%(n=9)$; TBT $101 \pm 16 \%$ $(n=10)$; MOT $69 \pm 16 \%(n=10)$; DPT $119 \pm 42 \%(n=8)$; DOT $115 \pm 33 \%(n=9)$; TPT $113 \pm 35 \%(n=10)$. Die Bestimmungsgrenzen (BG) des Verfahrens wurden nach DIN 32645 (1994) aus den Kalibrationsdaten (8 Niveaus) mit der Software SQS 2000 berechnet (Lernhardt und Kleiner 2000). Folgende BG wurden erreicht: $1 \mathrm{ng} / \mathrm{g}$ für MBT; $1-2 \mathrm{ng} / \mathrm{g}$ für TBT; je $2 \mathrm{ng} / \mathrm{g}$ für MOT, DOT und MPT; je $3 \mathrm{ng} / \mathrm{g}$ für DBT und DPT; $3-5 \mathrm{ng} / \mathrm{g}$ für TPT. Routinemäßig wurde jede Probe einmal aufgeschlossen und extrahiert, aber zweimal analysiert. Um die Wiederholbarkeit zu überprüfen, wurden Proben mehrfach untersucht $(n=3$; siehe Ergebnisse in Tabelle 1 und Tabelle 2). Die durchschnittliche relative Standardabweichung für die Mehrfachbestimmungen betrug für TBT $12 \%(n=9)$ und für TPT $23 \%(n=7)$. Insgesamt kann die Wiederholbarkeit als Maß für die Höhe der Messunsicherheit angesehen werden (die Richtigkeit wurde durch die Messung des CRM sowie die Wiederfindungsexperimente belegt).

\subsection{Trendprüfung}

Die Zeitreihen wurden mit dem nicht-parametrischen MannKendall-Test (Uhlig et al. 2002) auf statistisch signifikante Trends untersucht $(p<0,05)$; hierzu wurden Konzentrationen unterhalb der Bestimmungsgrenze auf $50 \%$ des Werts der jeweiligen Bestimmungsgrenze gesetzt.

\section{Ergebnisse und Diskussion}

\subsection{Monitoring-Ergebnisse}

Archivierte Biotaproben der Umweltprobenbank aus Nordund Ostsee wurden bereits früher auf Organozinnverbindun-

Tabelle 1 Ergebnisse Miesmuscheln, Angaben in ng OZK/g Frischgewicht; Werte unterhalb der BG sind mit < xx angegeben. Für DOT, MOT, DPT und MPT waren alle Werte unterhalb der BG

\begin{tabular}{|c|c|c|c|c|c|c|c|c|c|c|c|c|}
\hline \multirow{2}{*}{$\begin{array}{l}\text { Mies- } \\
\text { mu- } \\
\text { scheln }\end{array}$} & \multicolumn{4}{|c|}{ Nordsee, Eckwarderhörne (Jadebusen) } & \multicolumn{4}{|c|}{ Nordsee, Königshafen/Sylt } & \multicolumn{4}{|c|}{ Ostsee, Darßer Ort } \\
\hline & $\begin{array}{l}\text { TBT } \\
{[\mathrm{ng} / \mathrm{g} \mathrm{FG}]}\end{array}$ & $\begin{array}{l}\mathrm{DBT} \\
{[\mathrm{ng} / \mathrm{g} \mathrm{FG}]}\end{array}$ & $\begin{array}{l}\text { MBT } \\
{[\mathrm{ng} / \mathrm{g} \mathrm{FG}]}\end{array}$ & $\begin{array}{l}\text { TPT } \\
{[\mathrm{ng} / \mathrm{g} \mathrm{FG}]}\end{array}$ & $\begin{array}{l}\text { ТВT } \\
{[\mathrm{ng} / \mathrm{g} \mathrm{FG}]}\end{array}$ & $\begin{array}{l}\text { DBT } \\
{[\mathrm{ng} / \mathrm{g} \mathrm{FG}]}\end{array}$ & $\begin{array}{l}\text { MBT } \\
{[\mathrm{ng} / \mathrm{g} \text { FG] }}\end{array}$ & $\begin{array}{l}\text { TPT } \\
{[\mathrm{ng} / \mathrm{g} \mathrm{FG}]}\end{array}$ & $\begin{array}{l}\text { TВT } \\
{[\mathrm{ng} / \mathrm{g} \text { FG] }}\end{array}$ & $\begin{array}{l}\mathrm{DBT} \\
{[\mathrm{ng} / \mathrm{g} \mathrm{FG}]}\end{array}$ & $\begin{array}{l}\text { MBT } \\
{[\mathrm{ng} / \mathrm{g} \mathrm{FG}]}\end{array}$ & $\begin{array}{l}\text { TPT } \\
{[\mathrm{ng} / \mathrm{g} \mathrm{FG}]}\end{array}$ \\
\hline 2000 & 21 & 4 & 4 & 8 & 9 & $<3$ & 2 & 5 & 15 & $<3$ & 2 & $<3$ \\
\hline 2001 & 27 & 3 & 4 & 6 & $10 \pm 1^{*}$ & $<3$ & $2 \pm<1 *$ & $4 \pm<1^{*}$ & 15 & $<3$ & 2 & $<3$ \\
\hline 2002 & $24 \pm 3 *$ & $3 \pm 1 *$ & $4 \pm<1 *$ & $3 \pm 1 *$ & 8 & $<3$ & 2 & $<3$ & $14 \pm<1^{*}$ & $<3^{*}$ & $2 \pm<1 *$ & $<3 *$ \\
\hline 2003 & 23 & 4 & 4 & $<3$ & $9 \pm 2 *$ & $<3^{*}$ & $2 \pm<1^{*}$ & $<3^{*}$ & 10 & $<3$ & 2 & $<3$ \\
\hline 2004 & 14 & $<3$ & 3 & $<3$ & 3 & $<3$ & 2 & $<3$ & $5 \pm<1 *$ & $<3 *$ & $1 \pm<1 *$ & $<3 *$ \\
\hline 2005 & 6 & $<3$ & 3 & $<3$ & 3 & $<3$ & 2 & $<3$ & 4 & $<3$ & 1 & $<3$ \\
\hline BG & 2 & 3 & 1 & 3 & 2 & 3 & 1 & 3 & 2 & 3 & 1 & 3 \\
\hline
\end{tabular}

* Für diese Proben wurden je drei Replikate extrahiert und analysiert (angegeben als Mittelwert \pm Standardabweichung)

Tabelle 2 Ergebnisse Aalmuttermuskulatur, Angaben in ng OZK/g Frischgewicht; Werte unterhalb der BG sind mit < xx angegeben. Für DBT, DOT, MOT, DPT und MPT waren alle Werte unterhalb der BG

\begin{tabular}{|c|c|c|c|c|c|c|c|c|c|}
\hline \multirow{2}{*}{$\begin{array}{l}\begin{array}{l}\text { Aalmutter- } \\
\text { muskulatur }\end{array} \\
\text { Probenahmejahr }\end{array}$} & \multicolumn{3}{|c|}{$\begin{array}{c}\text { Nordsee, Transekt Varel-Mellum } \\
\text { (Jadebusen) }\end{array}$} & \multicolumn{3}{|c|}{ Nordsee, Meldorfer Bucht } & \multicolumn{3}{|c|}{ Ostsee, Darßer Ort } \\
\hline & $\begin{array}{l}\text { TBT } \\
{[\mathrm{ng} / \mathrm{g} \mathrm{FG}]}\end{array}$ & $\begin{array}{l}\mathrm{MBT} \\
{[\mathrm{ng} / \mathrm{g} \mathrm{FG}]}\end{array}$ & $\begin{array}{l}\text { TPT } \\
{[\mathrm{ng} / \mathrm{g} \mathrm{FG}]}\end{array}$ & $\begin{array}{l}\text { TBT } \\
{[\mathrm{ng} / \mathrm{g} \mathrm{FG}]}\end{array}$ & $\begin{array}{l}\mathrm{MBT} \\
{[\mathrm{ng} / \mathrm{g} \mathrm{FG}]}\end{array}$ & $\begin{array}{l}\text { TPT } \\
{[\mathrm{ng} / \mathrm{g} \mathrm{FG}]}\end{array}$ & $\begin{array}{l}\text { TBT } \\
{[\mathrm{ng} / \mathrm{g} \mathrm{FG}]}\end{array}$ & $\begin{array}{l}\mathrm{MBT} \\
{[\mathrm{ng} / \mathrm{g} \text { FG] }}\end{array}$ & $\begin{array}{l}\text { TPT } \\
{[\mathrm{ng} / \mathrm{g} \text { FG] }}\end{array}$ \\
\hline 1999 & 21 & $<1$ & 37 & 18 & 2 & 24 & 7 & $<1$ & 5 \\
\hline 2000 & 11 & $<1$ & 14 & 13 & 2 & 12 & 9 & $<1$ & 5 \\
\hline 2001 & $13 \pm 2 *$ & $<1^{*}$ & $12 \pm 1^{*}$ & 12 & 2 & 10 & $8 \pm<1^{*}$ & $<1 *$ & $5 \pm<1 *$ \\
\hline 2002 & 6 & $<1$ & 8 & $15 \pm 2 *$ & $1 \pm<1^{*}$ & $9 \pm<1 *$ & 10 & $<1$ & 3 \\
\hline 2003 & $10 \pm 1^{*}$ & $2 \pm 1^{*}$ & $9 \pm 1 *$ & 12 & 1 & 9 & $11 \pm 1^{*}$ & $<1^{*}$ & $<3 *$ \\
\hline 2004 & 9 & 2 & 7 & 9 & 1 & 3 & 5 & $<1$ & $<3$ \\
\hline 2005 & 6 & 3 & 7 & 7 & 1 & 4 & 5 & $<1$ & $<3$ \\
\hline 2006 & 6 & 1 & 4 & 5 & 1 & 4 & $<2$ & $<1$ & $<3$ \\
\hline BG & 2 & 1 & 3 & 2 & 1 & 3 & 2 & 1 & 3 \\
\hline
\end{tabular}

* Für diese Proben wurden je drei Replikate extrahiert und analysiert (angegeben als Mittelwert \pm Standardabweichung) 
gen untersucht (Rüdel et al. 2003). Dabei wurden TBT und TPT in fast allen Proben nachgewiesen. Eine Abnahme der TPT-Belastung wurde für Blasentang, Miesmuscheln und Aalmuttern für den Zeitraum 1985-1999 beobachtet. In dieser Zeit verringerten sich die TPT-Konzentrationen in den Nordseemuscheln von 98 auf 7 ng/g FG. Konzentrationen von TBT in Miesmuscheln blieben dagegen verhältnismäßig konstant; die Werte lagen bei $17 \pm 3 \mathrm{ng} / \mathrm{g}$ FG im Jadebusen, der durch den Verkehr großer Seeschiffe auf einer nahegelegenen Hauptschifffahrtsroute der Nordsee beeinflusst ist, bzw. bei $8 \pm 2$ ng/g FG nahe Sylt. Erwartungsgemäß wirkte sich in der Nordsee das auf kleine Schiffe $(<25 \mathrm{~m})$ beschränkte Verbot der TBT-Anwendung von 1989 nicht aus.

Im Rahmen der hier vorgestellten Untersuchungen wurden die entsprechenden Zeitreihen verlängert. Auf diese Weise sollte überprüft werden, ob die EU-Richtlinie 2002/62/EG (EU-Kommission 2002), die weitere Schritte zur Verminderung der Einträge von OZV in Gewässer regelt, eine Verringerung der Umweltkonzentrationen dieser Stoffe bewirkt. Wie Tabelle 1 und Abb. 1 zeigen, weisen die 2005 beprobten Miesmuscheln tatsächlich die niedrigsten OZVGehalte der jeweiligen Zeitreihe auf. Zuvor steigen die TBTGehalte im Zeitraum 2000-2003 aber sogar an (Abb. 1). Hier könnte eine Rolle spielen, dass in diesem Zeitraum, als schon über ein TBT-Verbot diskutiert wurde, Reedereien ihre Schiffe noch mit OZV-haltigen Antifouling-Anstrichen behandeln ließen, um die Wirkung möglichst lange zu sichern oder um Restbestände aufzubrauchen. Der Vergleich der drei Untersuchungsgebiete belegt, dass die OZV-Belastung der Muscheln aus dem Jadebusen am höchsten und die der nahe Sylt beprobten Muscheln am niedrigsten ist. Die Ostseemuscheln liegen in ihrer Belastung dazwischen. Die Zeitreihen wurden mittels Mann-Kendall-Test auf Trends untersucht. Während die TBT-Trends für den Gesamtzeitraum 1985-2001 nicht signifikant sind, ist die Abnahme im Zeitraum 2001-2005 signifikant ( $p<0,05$; für Muscheln aus dem Jadebusen und der Ostsee). Für die Muscheln aus dem Gebiet Königshafen nahe Sylt ist 2004 und 2005 ebenfalls ein deutlicher Rückgang der TBT-Gehalte zu erkennen, der aber noch nicht statistisch signifikant ist.

Die Konzentrationen von TPT in Muscheln sinken seit Mitte der 1980er-Jahre, nachdem es anscheinend nicht mehr als Co-Wirkstoff in Antifouling-Präparaten eingesetzt wurde (IKSR 2002; Rüdel et al. 2003). Die Abnahme der TPTKonzentration in Muscheln aus dem Jadebusen im Zeitraum 1985-2005 ist signifikant ( $p<0,05$; Abnahme von $98 \mathrm{ng} / \mathrm{g}$ FG in 1985 auf Werte <3 ng/g FG 2005), wie auch die - bei einem insgesamt niedrigeren Belastungsniveau - für den Nordseestandort nahe Sylt ( $p<0,05$; Abnahme von $20 \mathrm{ng} / \mathrm{g}$ FG in 1986 auf Werte $<3 \mathrm{ng} / \mathrm{g} \mathrm{FG}$ in 2005). Neben TBT und TPT sind noch MBT sowie in einzelnen Jahren DBT (Jadebusen) in niedrigen Konzentrationen nachweisbar.

Die Analysen von Aalmuttermuskulaturen bestätigen die Ergebnisse der Muscheluntersuchungen. Auch für Aalmuttern ist ein Rückgang der TBT- und TPT-Gehalte zu beobachten (Tabelle 2). Die TBT-Belastung der Aalmuttern an beiden Nordseestandorten ist ähnlich, während die Fische aus der Ostsee geringer belastet waren (insbesondere in den 1990er-Jahren; Abb. 2). Auch die TPT-Gehalte der Nordseeproben waren höher als in Ostsee-Fischen, wo in den letzten Jahren kein TPT mehr nachweisbar war. Die Abnahme der TBT- und TPT-Konzentrationen ist an allen UPB-Standorten statistisch signifikant $(p<0,05)$.

Im Gegensatz zu den Muscheln konnte in den Aalmuttermuskulatur-Proben DBT nicht nachgewiesen werden. Hier scheint somit eine schnelle Elimination oder eine direkte Metabolisierung zu MBT zu erfolgen. Generell ist die Metabolisierung von OZV in Fischen (vorwiegend in der Leber) offenbar höher als in Muscheln. So wurde in Fischen innerhalb von $48 \mathrm{~h}$ ein TBT-Abbau von bis zu $50 \%$ beobachtet, wobei DBT und MBT als Metaboliten identifiziert wurden. Dagegen war der Abbau in Muscheln deutlich geringer (Lee 1985; Fent 1996). DPT als potenzielles Abbauprodukt von TPT war nur in früheren Jahren in Aalmuttern und Miesmuscheln nachweisbar, als die TPT-Rückstände noch höher waren (Rüdel et al. 2003). Die hier gefundenen
Abb. 1 Zeitreihe der TBT-Gehalte in Miesmuscheln von den UPB-Standorten in Nord- und Ostsee. Daten aus dem Zeitraum vor dem Jahr 2000 stammen aus Rüdel et al. (2003). Die Probenahmen in der Ostsee erfolgten erst ab 1992

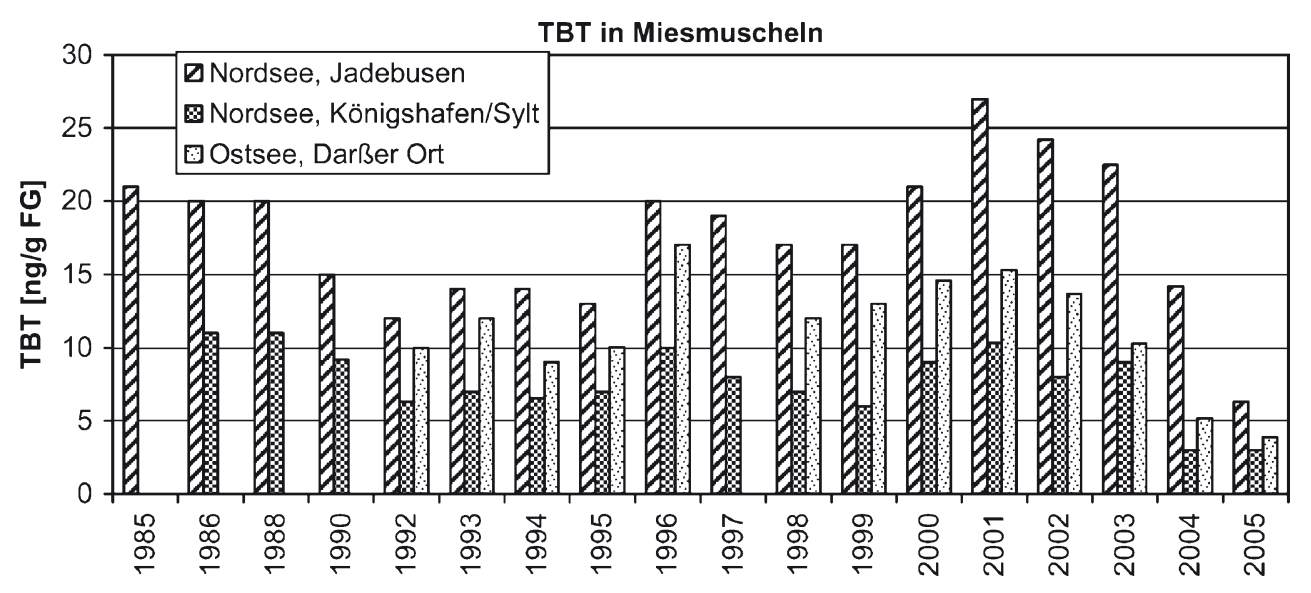


Abb. 2 Zeitreihe der TBT-Gehalte in Aalmuttern (Muskulatur) von den UPB-Standorten in Nord- und Ostsee. Daten aus dem Zeitraum vor dem Jahr 1999 stammen aus Rüdel et al. (2003). Proben aus der Meldorfer Bucht vor 1999 wurden nicht untersucht

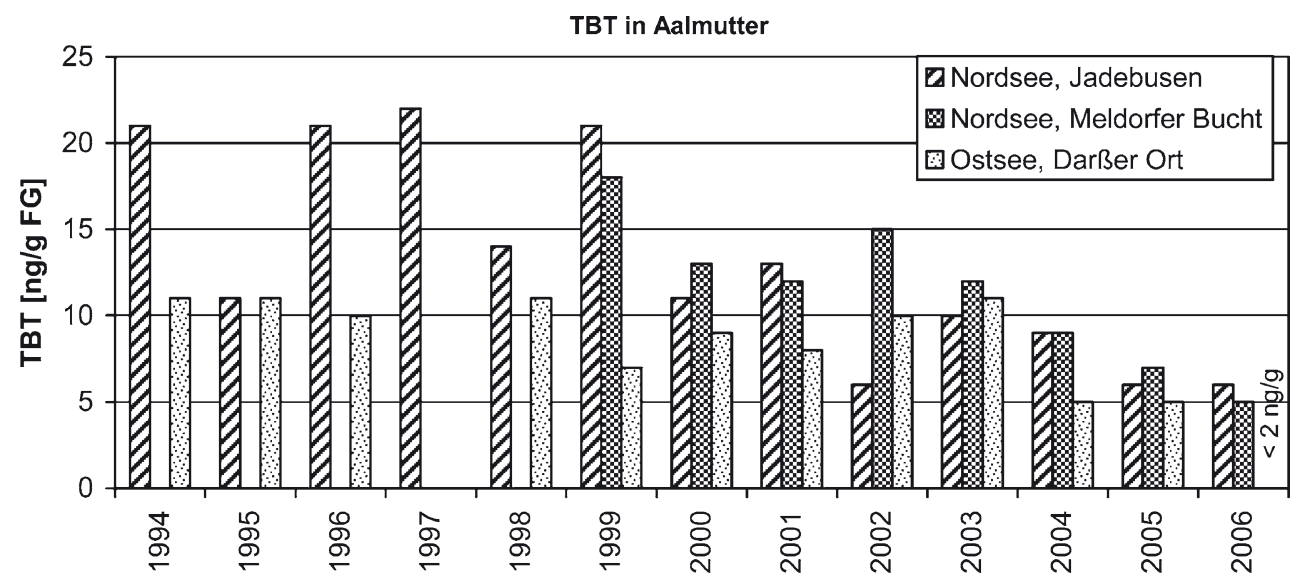

niedrigen Gehalte an TPT-Abbauprodukten werden durch Untersuchungen von Fent (1996) bestätigt, wonach bei der Exposition von Fischen mit TPT nur geringe Mengen der Abbauprodukte DPT und MPT gefunden wurden.

\subsection{Vergleich mit anderen Monitoring-Daten}

Zur Einordnung der aktuellen OZV-Belastung der Biotaproben aus Nord- und Ostsee wurde ein Vergleich mit anderen Monitoring-Daten für Biota aus europäischen Gewässern aus dem Zeitraum 2000-2005 durchgeführt (ein Vergleich der Ergebnisse bis 1999 mit anderen Literaturdaten geben Rüdel et al. 2003). Speziell für Butylzinnverbindungen hat kürzlich Antizar-Ladislao (2008) eine Übersicht publiziert. Generell wird in den dort ausgewerteten Studien von einem Rückgang der TBT-Belastungen berichtet. Höhere OZV-Konzentrationen bis zu maximal $790 \mathrm{ng} / \mathrm{g}$ TG bezogen auf Sn finden sich vor allem noch in Muscheln in der Nähe von Häfen und Werften. Dagegen sind in Regionen, die nicht oder nur wenig durch Schiffsverkehr beeinflusst sind, nur Konzentrationen im Bereich der analytischen Bestimmungsgrenze nachzuweisen. Für die hier untersuchten Proben aus dem UPB-Archiv lagen die höchsten Belastungen der vergangenen Jahre bei ca. $110 \mathrm{ng} / \mathrm{g}$ TG bezogen auf Sn für Muscheln (Nordsee, Jadebusen 2001) und ca. $25 \mathrm{ng} / \mathrm{g}$ TG bezogen auf Sn für Fische (Nordsee, Meldorfer Bucht, 2002).

Birchenough et al. (2002) untersuchten 1998 und 2000 die Butylzinn-Belastung von Neogastropoden sowie das Ausmaß an endokrinen Effekten (Imposex und Intersex) in verschiedenen Regionen der Nordsee. Dabei zeigte sich generell ein Rückgang der OZV-Belastung sowie auch der endokrinen Effekte im Vergleich zu zehn Jahren vorher. Höchste Belastungen und stärkste endokrine Effekte fanden sich an der englischen Küste in der Nähe von großen Werften. In der Nordseeregion, in der auch die UPB-Probenahmestellen liegen, wurden die Spezies Buccinum undatum und Neptunea antiqua auf hoher See beprobt. Hier waren sowohl die TBT-Belastung (im Bereich der Bestimmungsgrenze von $50 \mathrm{ng} / \mathrm{g}$ TG bzw. 2-5ng/g FG) als auch die Ausprägung von Imposex nur gering (unter $30 \%$ für B. undatum und unter $10 \%$ für $N$. antiqua; jeweils vorwiegend nur niedrigste Stufe der Imposex-Ausprägung).

Die ausgewerteten Literaturdaten bestätigen den Rückgang der OZV-Belastung in europäischen Gewässern in den vergangenen Jahren. Die OZV-Belastung der UPB-Probenahmegebiete in Nord- und Ostsee ist im Vergleich mit anderen Regionen eher als gering einzustufen. Dies ist wahrscheinlich darauf zurückzuführen, dass die Proben der UPB in Nationalparks gesammelt werden, die nur wenig von direkten Emissionen durch Häfen und Werften und nur mäBig durch Seeschiffsverkehr beeinflusst sind. Insbesondere das Ostsee-Probenahmegebiet im Bodden-Nationalpark ist weitgehend frei von potenziellen OZV-Quellen (im Gegensatz zu anderen Regionen der Ostsee).

\subsection{OZV-Einträge aus Flüssen}

Neben direkten Einträgen von OZV in die Meere, können insbesondere für die hier untersuchten Nordseegebiete auch Einträge aus Flüssen eine Rolle spielen. In einem Monitoring mit Süßwasserfischen waren im Rahmen der UPB ebenfalls OZV untersucht worden (Rüdel et al. 2007). Fast alle Proben aus Rhein, Elbe, Saale, Mulde und Saar wiesen Rückstände von TBT auf, mit überwiegend abnehmendem Trend. So waren für die im Rhein beprobten Brassen an allen Probenahmestellen signifikante Rückgänge der TBT-Konzentrationen zu beobachten (z.B. Koblenz und Weil: ca. $-85 \%$ ). Die weitaus höchsten TBT-Belastungen wurden in Brassen aus der Elbe nahe Blankenese nachgewiesen (185 ng/g FG im Jahr 2003). Hier ist der Einfluss des Schiffsverkehrs mit großen Frachtschiffen im Bereich des Hamburger Hafens zu erkennen. Mehr als $90 \%$ der TBT-Emissionen wird durch Schiffe verursacht, die länger als $100 \mathrm{~m}$ sind (IKSR 2002). Zusammengefasst belegte die Studie, dass TBT-Einträge aus Flüssen in die Nordsee in 
den letzten Jahren rückläufig sind. Ähnlich ist die Situation bei TPT. Die Belastung der untersuchten Fischproben nahm ebenfalls ab und trat in den letzten Jahren nur noch sporadisch auf. In den 1990er-Jahren korrelierten die Befunde mit der Verwendung von TPT als Fungizid in der Landwirtschaft, die 2001 mit der Rücknahme der Zulassung endete. Nur Elbe-Brassen, die bei Zehren gefangen wurden, zeigten danach noch höhere TPT-Gehalte (14 ng/g FG im Jahr 2003). Vor diesem Hintergrund ist der Eintrag von TPT über Flüsse in die Nordsee als Ursache der Belastung der marinen Proben wohl zu vernachlässigen.

\subsection{Abschätzung der Relevanz der OZV-Belastung des marinen Ökosystems}

Der Rückgang der OZV-Konzentrationen in den Geweben von marinen Organismen in den letzten Jahren ist deutlich. Von Interesse ist nun, ob die aktuell gemessenen Konzentrationen noch relevant sind hinsichtlich möglicher Effekte auf empfindliche Organismen. Hierzu wird mittels geeigneter Biokonzentrationsfaktoren (bioconcentration factor, BCF) von der Gewebekonzentration auf die mittlere Wasserkonzentration, der die Organismen ausgesetzt waren, umgerechnet (Tabelle 3). In der Ostsee lagen die so abgeschätzten OZV-Gehalte im Wasser im Jahr 2005 bei ca. 0,4-0,8 ng/1
TBT und $<0,1 \mathrm{ng} / 1$ TPT (auf Basis der Rückstände in Miesmuscheln) bzw. 0,5ng/1 TBT und $<1 \mathrm{ng} / 1 \mathrm{TPT}$ (auf Basis der Rückstände in Aalmuttermuskulatur). Für die Nordsee betragen die entsprechenden Werte $0,3-0,6$ ng/l TBT und $<0,1 \mathrm{ng} / 1 \mathrm{TPT}$ (Miesmuscheln) bzw. 0,5-0,7 ng/1 TBT und 1,0-2,3 ng/l TPT (Aalmutter). Während für TBT damit eine gute Übereinstimmung zwischen den aus Gewebekonzentrationen von Muscheln und Fischen berechneten Wasserkonzentrationen besteht, sind für TPT deutliche Unterschiede zu erkennen. Ursache hierfür könnte eventuell ein zu hoher BCF für Muscheln sein; da nur ein Wert für $M$. edulis verfügbar ist (Suzuki et al. 1998). Aber auch die unterschiedliche Exposition von Miesmuscheln und Aalmuttern könnte eine Rolle spielen. Da Aalmuttern sedimentbewohnende Fische sind, ist hier die Exposition vermutlich höher als für Muscheln (Desorption von sedimentgebundenem TPT). Insgesamt zeigen die Ergebnisse dieser Berechnungen, dass die Wasserkonzentrationen für TBT und TPT in der Nordsee durchaus noch in einem Bereich liegen, in dem Effekte an sensiblen Organismen beobachtet werden. So liegen die TBT-Konzentrationen oberhalb der nach EUWasserrahmenrichtlinie vorgeschlagenen UQN von $0,2 \mathrm{ng} / 1$ (EU-Kommission 2005).

Zur Umsetzung der OSPAR-Konvention (Oslo-ParisVertrag zum Schutz der marinen Umwelt im Nordost-At-

Tabelle 3 Umrechnung gemessener OZV-Gewebe-Konzentrationen auf Wasserkonzentrationen mittels Biokonzentrationsfaktoren (BCF)a Min./max.: minimaler/maximaler Wert der Umrechnung

\begin{tabular}{|c|c|c|c|c|c|c|c|}
\hline \multirow[t]{2}{*}{ Probenart/Probenahmestelle } & \multirow[t]{2}{*}{ Jahr } & \multirow{2}{*}{$\frac{\text { TBT }}{[n g / g F G]}$} & \multicolumn{2}{|c|}{ Berechnete Wasserkonzentration } & \multirow{2}{*}{$\frac{\text { TPT }}{[n g / g F G]}$} & \multicolumn{2}{|c|}{ Berechnete Wasserkonzentration } \\
\hline & & & $\begin{array}{l}{[\mathrm{ng} / 1] \text { TBT min. }} \\
B C F 5000\end{array}$ & $\begin{array}{l}{[\mathrm{ng} / \mathrm{l}] \mathrm{TBT} \max .} \\
B C F 10400\end{array}$ & & {$[\mathrm{ng} / \mathrm{l}] \mathrm{TPT} \min$.} & $\begin{array}{l}{[\mathrm{ng} / \mathrm{l}] \mathrm{TPT} \max .} \\
B C F 36000\end{array}$ \\
\hline \multirow[t]{3}{*}{ Nordsee, Transekt Varel-Mellum } & 2003 & 23 & 4,6 & 2,2 & $<3$ & - & $<0,1$ \\
\hline & 2004 & 14 & 2,8 & 1,3 & $<3$ & - & $<0,1$ \\
\hline & 2005 & 6 & 1,2 & 0,6 & $<3$ & - & $<0,1$ \\
\hline \multirow[t]{3}{*}{ Nordsee, Meldorfer Bucht } & 2003 & 9 & 1,8 & 0,9 & $<3$ & - & $<0,1$ \\
\hline & 2004 & 3 & 0,6 & 0,3 & $<3$ & - & $<0,1$ \\
\hline & 2005 & 3 & 0,6 & 0,3 & $<3$ & - & $<0,1$ \\
\hline \multirow[t]{3}{*}{ Ostsee, Darßer Ort } & 2003 & 10 & 2,0 & 1,0 & $<3$ & - & $<0,1$ \\
\hline & 2004 & 5 & 1,0 & 0,5 & $<3$ & - & $<0,1$ \\
\hline & 2005 & 4 & 0,8 & 0,4 & $<3$ & - & $<0,1$ \\
\hline Aalmuttermuskulatur & & & $B C F 9400$ & $B C F 11000$ & & $B C F 3100$ & $B C F 4100$ \\
\hline \multirow[t]{3}{*}{ Nordsee, Transekt Varel-Mellum } & 2004 & 9 & 1,0 & 0,8 & 7 & 2,3 & 1,7 \\
\hline & 2005 & 6 & 0,6 & 0,5 & 7 & 2,3 & 1,7 \\
\hline & 2006 & 6 & 0,6 & 0,5 & 4 & 1,3 & 1,0 \\
\hline \multirow[t]{3}{*}{ Nordsee, Meldorfer Bucht } & 2004 & 9 & 1,0 & 0,8 & 3 & 1,0 & 0,7 \\
\hline & 2005 & 7 & 0,7 & 0,6 & 4 & 1,3 & 1,0 \\
\hline & 2006 & 5 & 0,5 & 0,5 & 4 & 1,3 & 1,0 \\
\hline \multirow[t]{3}{*}{ Ostsee, Darßer Ort } & 2004 & 5 & 0,5 & 0,5 & $<3$ & $<1,0$ & $<0,7$ \\
\hline & 2005 & 5 & 0,5 & 0,5 & $<3$ & $<1,0$ & $<0,7$ \\
\hline & 2006 & $<2$ & $<0,2$ & $<0,2$ & $<3$ & $<1,0$ & $<0,7$ \\
\hline
\end{tabular}

a Bereich der BCF: 5.000-10.4001/kg für TBT in M. edulis (Laughlin et al. 1986; Suzuki et al. 1998) und 9.400-11.0001/kg in verschiedenen marinen Fischen (Yamada und Takayanagi 1992); 36.0001/kg für TPT in M. edulis (Suzuki et al. 1998) und 3.100-4.1001/kg in marinen Fischen (Yamada und Takayanagi 1992) 
lantik) wurden für wichtige Schadstoffe ökotoxikologische Bewertungskriterien (Ecotoxicological bzw. Environmental Assessment Criteria, EAC; OSPAR 1997; Moffat et al. 2004) abgeleitet. Als TBT-EAC für Muscheln wurde der Bereich 0,001-0,01 mg/kg Trockengewicht (OSPAR 1998) vorgeschlagen. Bei Überschreiten des unteren Werts besteht die Möglichkeit chronischer Effekte und bei Überschreiten des oberen Werts sind diese wahrscheinlich. Unter Berücksichtigung der Wassergehalte der hier untersuchten Muscheln (Nordsee/Königshafen 92,5 $\pm 1,5 \%$, Jadebusen $91,4 \pm 0,8 \%$, Ostsee $94,8 \pm 1,0 \%$ ) ergeben sich im Zeitraum 2003-2005 Konzentrationen von umgerechnet ca. $0,04-0,25 \mathrm{mg} / \mathrm{kg}$ TG in Muscheln aus dem Nordseeküstenbereich und ca. $0,08-0,20 \mathrm{mg} / \mathrm{kg}$ TG in Muscheln aus der Ostsee (die OSPAR-Konvention schließt die Ostsee allerdings nicht ein). Damit überschreiten auch die für die letzten Jahre nachgewiesenen TBT-Gehalte in Miesmuscheln den oberen EAC-Wert deutlich und chronische Effekte an Muscheln sind nicht auszuschließen. Derzeit findet eine Revision der TBT-EAC statt, und als neues Bewertungskriterium wurde ein Wert von 2,4 ng/g FG vorgeschlagen (Moffat et al. 2004; OSPAR 2005). Zumindest im Jahr 2005 lagen die TBT-Konzentrationen in Miesmuscheln sowohl in der Nord- als auch aus der Ostsee noch oberhalb dieses Wertes.

\section{Ausblick}

Die Ergebnisse dieser Studie belegen den Wert der Umweltprobenbank zur Überprüfung von Verwendungsbeschränkungen und $\mathrm{zu}$ Verboten von umweltrelevanten Chemikalien. Eine weitere Reduktion der Einträge von OZV in marine Ökosysteme ist zu erwarten, da zum 17. September 2008 eine im Rahmen der internationalen Seeschifffahrtsorganisation IMO (International Maritime Organisation) vereinbarte Konvention zum weltweiten Verbot von OZVhaltigen Antifouling-Produkten in Kraft getreten ist (AFS 2001: International Convention on the Control of Harmful Anti-Fouling Systems; IMO 2001). Um den Einfluss dieses Abkommens auf die OZV-Einträge in die Nord- und Ostsee zu untersuchen, sollte in den nächsten Jahren - neben dem inzwischen im Rahmen des OSPAR Coordinated Environment Monitoring Programme (CEMP) obligatorischen regelmäßigem Imposex-Monitoring - ein weiteres retrospektives Monitoring von TBT-Gehalten in marinen Biota im Rahmen der Umweltprobenbank durchgeführt werden. Idealerweise kann so der erwartete weitere Rückgang der OZV-Belastung in den Probenahmeregionen der Umweltprobenbank exemplarisch verifiziert werden.

Hierzu wäre allerdings die Etablierung eines neuen Analyseverfahrens notwendig, um niedrigere Bestimmungsgrenzen zu erreichen und die inzwischen abgesunkenen
Konzentrationen mit ausreichender Sicherheit quantifizieren zu können. In Frage käme insbesondere die speziesspezifische Isotopenverdünnungsanalytik mittels GC/ ICP-MS-Kopplung (Point et al. 2007). Durch Zugabe eines markierten Standards (z. B. ${ }^{119} \mathrm{Sn}$-markiertes TBT, DBT und MBT) ist das Zinnisotopenverhältnis nach dem Zeitpunkt der Addition und homogener Verteilung in der Aufschlusslösung weitgehend konstant, da sich die originär in der Probe vorhandenen und die zugegebene Butylzinnspezies nur noch über ihre Masse differenzieren lassen. Nach Ermittlung der resultierenden Verhältnisse der Isotope jeder Butylzinnspezies in der Probe und einem Vergleich mit den entsprechenden natürlichen Isotopenverhältnissen, kann dann eine sehr zuverlässige Gehaltsbestimmung durchgeführt werden.

Danksagung Besonderer Dank geht an die Kolleginnen und Kollegen der UPB-Teams des Instituts für Biogeographie (Universität Trier) für die Probennahmen sowie des Fraunhofer IME (Schmallenberg) für die Präparation der Jahreshomogenate. Herzlichen Dank auch an Dr. Martin Müller (Fraunhofer IME) für die Unterstützung bei der statistischen Analyse der Datensätze und Dr. Annette Fliedner (Fraunhofer IME) für die kritische Durchsicht des Manuskripts.

\section{Literatur}

Antizar-Ladislao B (2008) Environmental levels, toxicity and human exposure to tributyltin (TBT)-contaminated marine environment. A review. Environ Int 34(2):292-308

Arbeitskreis Umweltmonitoring (2007) Rüdel H, Bester K, Eisenträger A, Franzaring J, Haarich M, Köhler J, Körner W, Oehlmann J, Paschke A, Ricking M, Schröder W, Schröter-Kermani C, Schulze T, Schwarzbauer J, Theobald N, von der Trenck KT, Wagner G, Wiesmüller GA. Positionspapier zum stoffbezogenen Umweltmonitoring, Teil 1. Mitt Umweltchem Ökotox 13:34-41; Teil 2, Mitt Umweltchem Ökotox 13:72-79

Birchenough AC, Barnes N, Evans S M, Hinz H, Krönke I, Moss C (2002) A review and assessment of tributyltin contamination in the North Sea, based on surveys of butyltin tissue burdens and imposex/intersex in four species of neogastropods. Mar Pollut Bull 44(6):534-43

BMU (Bundesministerium für Umwelt, Naturschutz und Reaktorsicherheit) (2000) Umweltprobenbank des Bundes - Konzeption. Umweltbundesamt (Hrsg), Berlin. http://www.umweltbundesamt.de/ umweltproben/konzept/index.htm, Zugriff 11.03.2009

Böhmer W, Rüdel H, Wenzel A, Schröter-Kermani C (2004) Retrospective monitoring of triclosan and methyl-triclosan in fish: Results from the German environmental specimen bank. Organohalogen Comp 66:1516-1521

DIN 32645 (1994) Nachweis-, Erfassungs- und Bestimmungsgrenze - Ermittlung unter Wiederholbedingungen (Begriffe, Verfahren, Auswertung). Beuth Verlag, Berlin

EU-Kommission (2002) EU-Richtlinie 2002/62/EG der Kommission vom 9. Juli 2002 zur neunten Anpassung von Anhang I der Richtlinie 76/769/EWG des Rates zur Angleichung der Rechts- und Verwaltungsvorschriften der Mitgliedstaaten für Beschränkungen des Inverkehrbringens und der Verwendung gewisser gefährlicher Stoffe und Zubereitungen an den technischen Fortschritt (zinnorganische Verbindungen). Amtsblatt der Europäischen Gemeinschaften vom 12.7.2002. http://eur-lex.europa.eu/LexUriServ/site/de/oj/2002/ 1_183/1_18320020712de00580059.pdf, Zugriff 11.03.2009 
EU-Kommission (2005) Environmental Quality Standards (EQS). Substance Data Sheet Tributyltin compounds (TBT-ion) Priority Substance No. 30, CAS-No. 688-73-3 (36643-28-4), final version. Common Implementation Strategy for the Water Framework Directive. Brussels, 15 January 2005 http://circa. europa.eu/Public/irc/env/wfd/library?1=/framework_directive/ipriority_substances/supporting_background/substance_sheets/ eqsdatasheet $150105 \mathrm{pdf} 7 / \mathrm{EN} 1.0 \quad \& \mathrm{a}=\mathrm{d}$, Zugriff 11.03 .2009

EU-Richtlinie 2000/60/EG (2000) Richtlinie des Europäischen Parlaments und des Rates vom 23. Oktober 2000 zur Schaffung eines Ordnungsrahmens für Maßnahmen der Gemeinschaft im Bereich der Wasserpolitik. Amtsblatt Nr. L 327:0001-0073 vom 22/12/2000. http://eur-lex.europa.eu/LexUriServ/LexUriServ.do? uri=CELEX:32000L0060:DE:HTML, Zugriff 11.03.2009

Fent K (1996) Ecotoxicology of organotin compounds. Crit Rev Toxicol 26:1-117

Gibbs PE, Bryan GW (1996) TBT-induced imposex in neogastropod snails: masculinization to mass extinction. In: de Mora S J (ed) Tributyltin: Case Study of an Environmental Contaminant. Cambridge University Press, Cambridge, UK, pp 212-236

Graf GG (1996) Tin, Tin Aloys, and Tin Compounds. In: Ullmann's Encyclopedia of Industrial Chemistry, Vol. A27. VCH Verlagsgesellschaft, Weinheim, S 49-81

Hall Jr LW, Scott MC, Killen WD, Unger MA (2000) A probabilistic ecological risk assessment of tributyltin in surface waters of the Chesapeake Bay watershed. Hum Ecol Risk Assess 6(1):141-179

IKSR (Internationale Kommission zum Schutz des Rheins) (2002) Synthesebericht Antifoulings und Kühlwassersysteme. Bericht Nr. 132d.doc. IKSR, Koblenz. http://www.iksr.org/fileadmin/user_upload/ Dokumente/Berichte/bericht_132d.pdf, Zugriff 11.03.2009

IMO (International Maritime Organisation) (2001) International Convention on the Control of Harmful Anti-fouling Systems on Ships. Adoption: 5 October 2001. Entry into force: 17 September 2008. http://www.imo.org/conventions/mainframe.asp?topic_id=529, Zugriff 11.03.2009

Klein R, Paulus M, Wagner G, Müller P (1994) Das ökologische Rahmenkonzept zur Qualitätssicherung in der Umweltprobenbank des Bundes. UWSF - Z Umweltchem Ökotox 6: 223-231

Koglin D, Backhaus F, Schladot JD (1997) Particle size distribution in ground biological samples. Chemosphere 34:2041-2047

Laughlin RB, French W, Guard HE (1986) Accumulation of bis(tributyltin)oxide by the marine mussel Mytilus edulis. Environ Sci Technol 20:884-890

Lee RF (1985) Metabolism of tributyltin oxide by crabs, oysters, and fish. Mar Environ Res 17:145-148

Lernhardt U, Kleiner J (2000) SQS2000, Software für die statistische Kontrolle analytischer Daten und Methodenvalidierung, Version 1.1, Perkin Elmer Deutschland. Technische Schule, Überlingen

Moffat CF, Pijnenburg J, Trass T (eds) (2004) OSPAR/ICES Workshop on the evaluation and update of Background Reference Concentrations (BRCs) and Ecotoxicological Assessment Criteria (EACs) and how these assessment tools should be used in assessing contaminants in water, sediment, and biota. Report of the joint OSPAR/ ICES Workshop, 9-13 February 2004, The Hague, Netherlands

OSPAR (1997) OSPAR Agreement 1997-15. Agreed Ecotoxicological Assessment Criteria for Trace Metals, PCBs, PAHs, TBT and some Organochlorine Pesticides. http://www.ospar.org/documents/ dbase/decrecs/agreements/97-15e.doc, Zugriff 11.03.2009
OSPAR (1998) Report of the 3rd OSPAR Workshop on Ecotoxicological Assessment Criteria, Part III, 25.-29. November 1996, The Hague. OSPAR Secretariat, London, UK

OSPAR (2005) Assessment of data collected under the Co-ordinated Environmental Monitoring Programme (CEMP). http://www.ospar.org/ documents/dbase/publications/p00235_CEMP\%20report.pdf, Zugriff 11.03.2009

Point D, Davis WC, Christopher SJ, Ellisor MB, Pugh RS, Becker PR, Donard OFX, Porter BJ, Wise SA (2007) Development and applicaton of an ultratrace method for speciation of organotin compounds in cryogenically archived and homogenized biological materials. Anal Bioanal Chem 387:2343-2355

Rüdel H (2003) Case study: bioavailability of tin and tin compounds. Ecotoxicol Environ Saf 56(1):180-189

Rüdel H, Böhmer W, Schröter-Kermani C (2006) Retrospective monitoring of synthetic musk compounds in aquatic biota from German rivers and coastal areas. J Environ Monit 8:812-823

Rüdel H, Lepper P, Steinhanses J, Schröter-Kermani C (2003) Retrospective monitoring of organotin compounds in marine biota from 1985 to 1999: Results from the German environmental specimen bank. Environ Sci Technol 37:1731-1738

Rüdel H, Müller J, Steinhanses J, Schröter-Kermani C (2007) Retrospective monitoring of organotin compounds in freshwater fish from 1988 to 2003: Results from the German environmental specimen bank. Chemosphere 66:1884-1894

Schulte-Oehlmann U, Tillmann M, Markert B, Oehlmann J, Watermann B, Scherf S (2000) Effects of endocrine disruptors on prosobranch snails (Mollusca: Gastropoda) in the laboratory. Part II: triphenyltin as a xeno-androgen. Ecotoxicol 9:399-412

Suzuki T, Yamamoto I, Yamada H, Kaniwa N., Kondo K, Murayama MJ (1998) Accumulation, metabolism, and depuration of organotin compounds in the marine mussels Mytilus graynus and Mytilus edulis under natural conditions. J Agric Food Chem 46:304-313

UBA (Umweltbundesamt) (1996) Umweltprobenbank des Bundes - Verfahrensrichtlinien für Probenahme, Transport, Lagerung und chemische Charakterisierung von Umwelt- und HumanOrganproben. Umweltbundesamt (Hrsg), Erich Schmidt Verlag, Berlin

Uhlig S, Simon K, Schick N, Köhler S, Rothmaler D (2002) Entwicklung statistischer Verfahren für Ringversuche, Methodenvalidierung und Auswertung von marinen Daten - Teilvorhaben II. Im Auftrag des Umweltbundesamtes. quo data - Gesellschaft für Qualitätsmanagement und Statistik mbH, Texte 35/02, Umweltbundesamt, Berlin. http://www.umweltdaten.de/publikationen/ fpdf-1/2152.pdf, Zugriff 11.03.2009

Watermann B, Siebert U, Schulte-Oehlmann U, Oehlmann J (2003) Endokrine Effekte durch Tributylzinn (TBT). In: Lozan JL, Rachor E, Reise K, Sündermann J, v Westernhagen H. (Hrsg) Warnsignale aus Nordsee \& Wattenmeer. Eine aktuelle Umweltbilanz. Universität Hamburg, S 239-247

Wenzel A, Böhmer W, Müller J, Rüdel H, Schröter-Kermani C (2004) Retrospective monitoring of alkylphenols and alkylphenol monoethoxylates in aquatic biota from 1985 to 2001: Results from the German environmental specimen bank. Environ Sci Technol 38:1654-1661

Yamada H, Takayanagi K (1992) Bioconcentration and elimination of bis(tributyltin)oxide (TBTO) and triphenyltin chloride (TPTC) in several marine fish species. Water Res 26:1589-1595 